\title{
Microarray Based Functional Analysis of Myricetin and Proteomic Study on Its Anti-Inflammatory Property
}

\author{
Tao Li $\left(\mathbb{D},{ }^{1}\right.$ Jihe Zhu $\left(\mathbb{D},{ }^{1}\right.$ Fangming Deng, ${ }^{1}$ Weiguo Wu, ${ }^{1}$ Zhibing Zheng, ${ }^{1}$ Chenghao Lv, \\ Yong Li, ${ }^{1}$ Wei Xiang, ${ }^{2}$ Xiangyang Lu $\bullet{ }^{1,2}$ and Si Qin $\circledast^{1,2,3}$ \\ ${ }^{1}$ Core Research Program 1515, Key Laboratory for Food Science and Biotechnology of Hunan Province, \\ College of Food Science and Technology, Hunan Agricultural University, Changsha 410128, China \\ ${ }^{2}$ Hunan Co-Innovation Center for Utilization of Botanical Functional Ingredients, College of Bioscience and Biotechnology, \\ Hunan Agricultural University, Changsha 410128, China \\ ${ }^{3}$ The United Graduate School of Agricultural Sciences, Faculty of Agriculture, Kagoshima University, Korimoto 1-21-24, \\ Kagoshima 890-0065, Japan \\ Correspondence should be addressed to Xiangyang Lu; xiangyangcn@163.com and Si Qin; qinsiman@hunau.edu.cn
}

Received 13 November 2018; Accepted 11 February 2019; Published 7 March 2019

Guest Editor: Deguang Song

Copyright (C) 2019 Tao Li et al. This is an open access article distributed under the Creative Commons Attribution License, which permits unrestricted use, distribution, and reproduction in any medium, provided the original work is properly cited.

\begin{abstract}
Myricetin has been reported as a promising chemopreventive compound with multiple biofunctions. To evaluate its influence on gene expressions in genome-wide set and further investigate its anti-inflammatory property, the present study performed Gene Ontology and Ingenuity Pathway Analysis (IPA) to describe the basic gene expression characteristics by myricetin treatment in HepG2 cells, confirmed its multi-biofunction by real-time fluorescent quantitative PCR (RT-qPCR), and further verified its antiinflammatory property by Western blotting and bio-plex-based cytokines assay. The IPA data showed that 337 gene expressions ( $48 \%$ of the top molecules) are disturbed over 2-fold, and the most possible biofunctions of myricetin are the effect on "cardiovascular disease, metabolic disease, and lipid metabolism," via regulation of 28 molecules with statistic score of 46 . RT-qPCR data confirmed the accuracy of microarray data, and cytokines assay results indicated that 6 of the total 27 inflammatory cytokine secretions were significantly inhibited by myricetin pretreatment, including TNF- $\alpha$, IFN- $\gamma$, IL- $1 \alpha$, IL- $1 \beta$, IL-2, and IL- 6 . The present study is the first time to elucidate the multi-function of myricetin in genome-wide set by IPA analysis and verify its anti-inflammatory property by proteomics of cytokines assay. Therefore, these results enrich the comprehensive bioactivities of myricetin and reveal that myricetin has powerful anti-inflammatory property, which provides encouragement for in vivo studies to verify its possible health benefits.
\end{abstract}

\section{Introduction}

Myricetin is a well-defined natural flavonoid with hydroxyl groups at the positions of 3, 5, 7, 3', 4', and 5', which is widely existed in vegetables, fruits, and teas, as well as medicinal herbs [1]. Myricetin has been proved to be the most potent and promising chemopreventive compound for its multiple biofunctions, such as antioxidation, anti-inflammation, antitumor, anti-diabetes, and anti-mutation effects $[2,3]$. For the antioxidant property, myricetin was reported to attenuate the deleterious effect of oxidative stress in human red blood cells [4], prevent against I/R-induced myocardial injury in rats [5], and exhibit a significant hepatoprotective activity to reduce hepatic oxidative stress in mice [6], by the induction of antioxidant genes or proteins. For the antiinflammatory property, overexpression of tumor necrosis factor-alpha (TNF- $\alpha$ ) and cyclooxygenase-2 (COX-2) in mouse liver was found to be reduced by myricetin treatment [6], and the production of proinflammatory mediators was inhibited by myricetin via inhibition of NF- $\kappa$ B and STAT1 pathways and induction of Nrf2-HO-1 pathway in LPSstimulated RAW264.7 macrophages [7]. For the anticancer property, myricetin was recognized to be able to induce apoptosis of Human T24 bladder cancer cells via modulation of Bcl-2 family proteins and caspase-3, significantly inhibit the tumor growth on T24 bladder cancer xenografts model [8], and promote apoptosis through regulation of apoptotic protein Bax, Bad, and Bcl-2 in HepG2 cells [9]. 
The recent arising concept of nutrigenomics is defined to investigate the omics-wide influences of classical nutrients or other dietary bioactive components in food. Nutrients or dietary bioactive components are mediators that can be detected by the cellular sensor systems and influence gene expressions, protein synthesis, and metabolite production [10]. Nutrigenomics is designed to investigate the dietary components in distinguished cells, tissues, and organisms and to elucidate how they influence the redox balance and homeostasis of the cells, which plays crucial role on human health maintenance and disease prevention $[10,11]$. However, limited researches were performed to study the functional effects of flavonoids and elucidate the underlying mechanism by using methods of nutrigenomics. For instance, by using quantitative proteomics approach, EGCG was reported to exert its effect on alleviation to vanadium stress in laying hens through regulation of metal-binding mediation, cell proliferation, and immune function-related proteins [12]. Grape polyphenols were found to improve cellular parameters and reduce the amount of lipogenesis and glycolysis enzymes, enhance fatty acid oxidation and stimulate insulin signaling, and ameliorate protein oxidation or endoplasmic reticulum stress [13]. By using transcriptomics expression analysis, cocoa polyphenols were reported to possess antiobesity effects in high fat diet induced obese rats by regulation of lipid metabolism genes and reduce adiposity in adipose tissue [14]. Recently, a comprehensive review had summarized the data in the last decade and found that dietary polyphenols may function as ideal modulators of the mammalian gene expressions by histone deacetylation, histone acetylation, and DNA methylation in experimental models $[15,16]$. Another interesting review further provides the reliable scientific data to reveal the importance of polyphenols to fight against carcinogenesis epigenetically and focuses on the effects of dietary polyphenols to mitigate carcinogenesis [17]. Our previous study had applied microarray to analyze the effects of myricetin on genome-wide set; however, the analysis of the data remains scarce and shallow [18]. Therefore, further analysis on the microarray data of myricetin and other attached new omics tools should be performed, such as proteomics assay.

The studies on the biofunction of polyphenols mainly focus on the regulation of redox signaling pathways and related genes or proteins linked to human health, especially on the inhibition of inflammation [19]. Inflammation is defined by the increased acute phase reactants or other mediators, the activation of inflammatory signal pathways, and abnormal cytokine or inflammatory marker expressions [20]. Cytokines are important inflammatory signaling proteins to mediate a wide range of physiological responses and a spectrum of related diseases, such as tumor growth, infections, and Parkinson's disease. Cytokines are generally detected by either immunoassay or bioassay [21, 22]. Recently, the newly discovered Bio-Plex suspension array instrument from Bio-Rad Company can simultaneously detect up to 100 cytokines in a single well of a 96-well microplate by application of novel technology with color-coded beads. Therefore, bio-plex based technology is of great significance to understand the whole inflammatory process. To elucidate the more detail about the multiple biofunctions of myricetin, comprehensive analysis of microarray data in genome-wide set by advanced software and specific verification of the antiinflammatory property by proteomic tool were performed in the present study.

\section{Materials and Methods}

2.1. Materials and Antibodies. Myricetin ( $\geq 99 \%$, purified by HPLC) was purchased from Extrasynthese (Lyon Nord, France). Human hepatoblastoma (HepG2) cells were donated by the Cancer Cell Repository (Tohoku University, Japan). Fetal bovine serum (FBS) and Dulbecco's modified Eagle's medium (DMEM) were purchased from Hyclone (Logan, USA). Lipopolysaccharide (LPS) and other general reagents used in the chemical analysis were purchased from SigmaAldrich (Shanghai, China). The antibodies for COX-2, iNOS, $\alpha$-tubulin, and others were purchased from Santa Cruz Biotechnology (Santa Cruz, USA) and Cell Signaling Technology (Beverly, USA).

2.2. Reverse Transcription and Real-Time qPCR. HepG2 cells were planted in dishes for $24 \mathrm{~h}$ and then treated with $20 \mu \mathrm{M}$ of myricetin in $0.1 \%$ DMSO or alone for additional $9 \mathrm{~h}$. Total RNA was extracted with RNA extraction kit (Nippon Gene Co., Japan) as described accordingly. The primers used in the present study were designed by PRIMER3 and synthesized by company as follows: AKR1C1, forward (5'- ATC CCT CCG AGA AGA ACC AT-3') and reverse (5'- ACA CCT GCA CGT TCT GTC TG-3'); AKR1C2, forward (5'- GAT CCC ATC GAG AAG AAC CA-3') and reverse (5'-ACA CCT GCA CGT TCT GTC TG-3'); GCLC, forward (5'- GAG CTG GGA GGA AAC CAA G -3') and reverse (5'- TGG TTT GGG TTT GTC CTT TC-3'); SERPINE, forward (5'GTG CTG GTG AAT GCC CTC T-3') and reverse (5’- GCA GTT CCA GGA TGT CGT -3'); IL11, forward (5'-GCG GAC AGG GAA GGG TTA AAG-3') and reverse (5'- GGG CGA CAG CTG TAT CTG G -3'); IGFBP1, forward (5' - ATG ATG GCT CGA AGG CTC TC-3') and reverse (5'-ATG TCT CAC ACT GTC TGC TGT-3'). Reverse transcription and realtime qPCR were performed with RT-qPCR Kit (Finnzymes Oy., Espoo, Finland) accordingly. Briefly, $200 \mathrm{ng}$ of RNA was reverse-transcribed to $\mathrm{cDNA}$ at $37^{\circ} \mathrm{C}$ for $30 \mathrm{~min}$, and the reaction was then terminated at $85 \circ \mathrm{C}$ for $5 \mathrm{~min}$, and other reaction conditions or procedures were described previously [18]. The results were presented as the relative expression levels normalized with that in control cells.

\subsection{Microarray Data Analysis and Network Generation.} Microarray results were classified by Gene Ontology ID (http://www.geneontology.org/) and analyzed by general method and then were imported into Ingenuity Pathway Analysis (IPA) System (http://www.ingenuity.com) for further analysis. The gene ontology classification and analysis are primary bioinformatics to standardize their presentation of gene and gene product attributes from different species and databases. The Ingenuity Knowledge Base is built by the huge data extracted from the millions of full text literatures 
with weekly update, which is the leader of its kind to analyze the results originated from microarray. [23]. The original microarray data was imported into the IPA system according to gene accession numbers and the fold change upon myricetin treatment versus control and other necessary data. $\mathrm{P}<0.002$ was set as the cutoff point of the IPA system, and the genes were classified according to the molecular functions. The canonical pathways analysis was performed to identify the most possible disturbed pathways from the IPA system with the most significance in the dataset [24, 25].

The network analysis was carried out to identify series of genes belonging to certain genetic networks associated with functions or diseases in the IPA system and were ranked by the score that represents the possibility that a class of genes were disturbed. The genetic network analysis was also based on the information or findings originated from millions of literatures focused on the study of functional relationships between genes. A network pathway contains a class of genes or gene products, which are represented as nodes, and the biological relationship between 2 nodes is represented as an edge (line). This kind of network is a graphical representation of the molecular relationships between genes or gene products. All the correlations or links are supported by at least one reference stored in the Ingenuity Pathways Knowledge Base, including literature, textbook, and canonical information. The intensity of the node color indicates the degree of regulation: the red represents up and the green represents down. The various shapes of nodes and labels of edges describe different functional gene product and the nature of the relationship between the nodes, respectively.

2.4. Immunoblots of Inflammatory Proteins. HepG2 cells were precultured in dishes for $24 \mathrm{~h}$ first and then starved by adding serum-free medium for additional $2.5 \mathrm{~h}$. The cells were divided into 4 groups, which were a control group without treatment; a negative control group treated with 1 $\mu \mathrm{g} / \mathrm{ml}$ of LPS alone; two groups were treated with $20 \mu \mathrm{M}$ of myricetin before exposure to LPS, or not. Every group were treated for $30 \mathrm{~min}$. Cellular lysates were boiled for $5 \mathrm{~min}$, equal amounts of which $(40 \mu \mathrm{g})$ were run on SDS-PAGE gel $(10 \%)$ and transferred to PVDF membrane (Amersham Pharmacia Biotech). The membrane was incubated with specific primary antibody overnight at $4^{\circ} \mathrm{C}$ and incubated with respective secondary antibodies for $1 \mathrm{~h}$. Immunoblot binds were detected by ECL system with the Image Quant LAS 4000 mini (GE Healthcare, Chicago, US). The relative amount of detected proteins was quantified by ImageQuant TL software.

2.5. Bio-Plex-Based Assays of Inflammatory Cytokine. HepG2 cells were precultured and starved accordingly to eliminate the effect of FBS. The cells were then treated with 10-20 $\mu \mathrm{M}$ of myricetin for half hour before exposure to $1 \mu \mathrm{g} / \mathrm{mL}$ LPS for additional $12 \mathrm{~h}$. The 27 cytokines detection, including TNF- $\alpha$, G-CSF, IFN- $\gamma$, IL- $1 \alpha$, IL-1 $\beta$, IL-2, IL-4, IL-5, IL-6, IL7, IL-8, IL-9, IL-10, IL-12(p70), IL-13, IL-15, IL-17, RANTES, Eotaxin, PDGF-BB, FGF basic, IP-10, MCP-1(MCAF), MIP$1 \alpha$, MIP-1 $\beta$, GM-CSF, and VEGF were performed by using
Bio-Plex Pro Human Cytokine 27-Plex Panel kit (Bio-Rad Laboratories) and Bio-Plex cytokines assay system (Bio-Plex 200, Bio-Rad) according to the manufacturer's instructions and the results were analyzed by the Bio-Plex manager software (version 4.0).

2.6. Statistical Analysis. All the experimental data in the present study were repeated at least three or four times. Significances or differences of treated versus control were analyzed by the Student's t-test, and $p<0.05$ was considered significant.

\section{Results}

3.1. Gene Expression Profiling of Myricetin-Treated HepG2 Cells by $G O$ Analysis. According to our previous study, we have performed basic analysis to the huge microarray data. Among the total $44 \mathrm{~K}$ gene probes, $20 \mu \mathrm{M}$ of myricetin upregulated the expressions of 143 genes $(0.33 \%$ of total probes) and downregulated the expressions of 476 genes (1.08\% of total probes) by greater than or equal to twofolds in HepG2 cells [18]. However, the comprehensive analysis of the data is scarce. Thus, we further utilize the free available tool, Gene Ontology, to analyze the basic characteristic of gene expressions in HepG 2 cells by myricetin treatment. As shown in Table 1, three basic classes, including biological process, molecular function, and cellular component, were list out. In each class, we further list out the gene function subclasses with the ratio of significant regulated genes greater than $2 \%$ of the total, which includes 3, 5, and 16 subclasses, respectively.

Among biological process, signal transduction, metabolism, and lipid metabolism have been largely disturbed by myricetin treatment, with significantly regulated gene ratio of $7.27 \%, 4.68 \%$, and $2.26 \%$, respectively, which implies that myricetin has the potency on the regulation of signaling transduction and cellular metabolism, especially lipid metabolism. For instance, in signal transduction, expressions of F2RL2, IGFBP1, ARHGAP26, PDE11A, ADM, SOS1, NF1, F2RL1, IL8, VDR, and TXNRD1 were identified to be linked to the biofunction of myricetin (Table S1). In cellular component, one of the notable affected subclasses is nucleus, containing the largest gene ratio of $19.22 \%$. It involves several response element promoters of signaling pathways, such as ARE, NF- $\kappa \mathrm{B}, \mathrm{XRE}$, or AP-1, which are associated with oxidative stress response, inflammation, or xenobiotic metabolism. There are several gene expressions were remarkably altered in cellular component, including BHLHB2, DIDO1, NDRG1, EID3, SORBS2, WDHD1, KIAA1429, SORBS2, JUN, and SLC2A4RG, as shown in Table $\mathrm{S} 2$. Another important subclass is mitochondrion, with the differential gene ratio of $4.2 \%$. Mitochondrion is critical in endothelial physiology and pathophysiology, and plays a prominent role on production of ATP, energy currency of the cell, through respiration, and then regulates the cellular metabolism [26, 27]. Myricetin was found to exert a crucial role on mitochondria function and keep the redox balance, which was evidenced by the differentially expressed genes after myricetin medication, such as HSPA1A, GLS, 
TABLE 1: Gene expression profiling by GO analysis.

\begin{tabular}{lcc}
\hline Class & Subclass & Ratio [\%] \\
\hline \multirow{3}{*}{ Biological process } & Signal transduction & 7.27 \\
& Metabolism & 4.68 \\
& Lipid metabolism & 2.26 \\
\hline Cellular component & Nucleus & 19.22 \\
& Extracellular & 7.27 \\
& Plasma membrane & 6.79 \\
& Cytosol & 3.72 \\
& Mitochondrion & 4.20 \\
\hline & Protein binding & 27.30 \\
& DNA binding & 10.02 \\
& Binding & 7.59 \\
& Kinase & 3.39 \\
& Protein kinase activity & 2.26 \\
& calcium ion binding & 3.88 \\
& nucleic acid binding & 6.46 \\
& transcription factor activity & 4.36 \\
Molecular function & RNA binding & 3.55 \\
& nucleotide binding & 9.69 \\
& actin binding & 3.55 \\
& catalytic activity & 5.33 \\
& Receptor activity & 2.26 \\
& protein kinase activity & 2.26 \\
& hydrolase & 5.98 \\
& & 5.65 \\
\hline
\end{tabular}

TABLE 2: Gene expression profiling of myricetin-treated HepG2 cells by IPA analysis.

\begin{tabular}{|c|c|c|c|}
\hline \multirow{2}{*}{ Fold of Change } & \multirow[b]{2}{*}{ Total } & \multicolumn{2}{|c|}{ IPA data } \\
\hline & & Numbers & Regulation \\
\hline \multirow{2}{*}{$>4$} & \multirow{2}{*}{15} & 8 & up \\
\hline & & 7 & down \\
\hline \multirow{2}{*}{$3<\sim<4$} & \multirow{2}{*}{36} & 16 & up \\
\hline & & 20 & down \\
\hline \multirow{2}{*}{$2<\sim<3$} & \multirow{2}{*}{286} & 56 & up \\
\hline & & 230 & down \\
\hline \multirow{2}{*}{ Total } & \multirow{2}{*}{337} & 80 & up \\
\hline & & 257 & down \\
\hline
\end{tabular}

PDK1, ABAT, SYNE2, ATP5S, and ETFDH (Table S3). The significantly activated molecular functions were generally related to protein binding, DNA binding, kinase, and protein kinase activity, with the corresponding proportion of $27.3 \%$, $10.02 \%, 3.39 \%$, and $2.26 \%$, respectively. Almost all the features of cells on their surfaces and interiors are based on diversity protein carrier in terms of tool-like receptor, facilitated glucose transporter, thioredoxin reductase 1, etc. Before that, many signal pathways associated to molecular function rely on DNA linking, the deliverance of genetic information, and the period is usually activated by protein kinases [28]. As shown in Table S3, a series of typical genes belonging to the above 4 subclasses disturbed by myricetin have been listed out, such as SLC2A4RG, SLC22A3, SERPINE1, MAP3K13, ACVR1B, MAP3K8, ARHGAP26, BHLHB2, DMXL1, NCF2, HSPA1A/B, HMOX1, FGFR3, ATR, FGFR3, ACVR1B, WDHD1, HELLS, SOS1, and JUN. These results indicated that myricetin has the potential to regulate molecular function, via regulation of gene expression pathways and modulation of signal transduction.

3.2. Gene Expression Profiling of Myricetin-Treated HepG2 Cells by IPA Analysis. Next, to further analyze the microarray data, we input the data into IPA system, and the gene expression profiling of myricetin-treated HepG2 cells has changed a little. As shown in Table 2, comparing the signals of myricetin-treated group with the control, the result revealed that the expressions of 15 genes were disturbed significantly by equal or greater than 4 -fold, with upregulation of 8 genes and downregulation of 7 genes; the expressions of 36 genes were disturbed between 3 -fold to 4 -fold, with upregulation of 16 genes and downregulation of 20 genes; the expressions of 286 genes were disturbed between 2 -fold to 3fold, with upregulation of 56 genes and downregulation of 230 genes. Taken together, 337 gene expressions of the total 702 molecules $(48 \%)$ exported by IPA were disturbed with fold changes over 2-fold.

3.3. Top Function and Canonical Pathway Analysis of Myricetin-Treated HepG2 Cells by IPA. To obtain a further investigation for the elucidation of the effects and underlying molecular mechanism of myricetin in HepG2 cells, the advanced analysis tool of IPA system was performed. In brief, IPA constructed the connection and relationship of the above significantly regulated genes, which also output the top 10 cellular functions and diseases altered by myricetin treatment in HepG2 cells with a $p$-value less than 0.05 , as shown in Table 3 . The most possible cellular function/disease of myricetin is the regulation of "cardiovascular disease, metabolic disease, and lipid metabolism," which consists of 28 molecules with the score of 46 . The secondary top cellular function/disease, "connective tssue development and function, skeletal and muscular system development and function, and tissue morphology" was significantly influenced by myricetin treatment as well, with 22 molecules and the score of 33 . Besides, other cellular functions/diseases, such as "endocrine system disorders," "cardiovascular system development and function," "cellular assembly and organization," "cellular movement," "cancer," "cell death," and "hepatic system disease," were also identified to be significantly disturbed by myricetin treatment. However, "cancer" was found to be the most frequently influenced disease with the occurrence of 4 times in top 10 functions, and "cell death" and "metabolism disease" were both mentioned 3 times there. The above result indicates that myricetin may exert its biofunction by the regulation of chronic disease related biomarkers and the representative signaling pathways. For instance, MAP3K8, MAP3K13, NCF2, $N F-\kappa B$ (family), NFATC2, NF- $\kappa B$ (complex), and NR2F2 were significantly disturbed by myricetin treatment, which are belonging to cancer related MAPK/NF- $\kappa \mathrm{B}$ inflammatory signaling pathway. 
TABLE 3: Top 10 cellular functions and diseases altered by myricetin treatment in HepG2 cells.

\begin{tabular}{l}
\hline \\
\hline Cardiovascular Disease, Metabolic \\
Disease, Lipid Metabolism
\end{tabular}

Cardiovascular Disease, Meta
Disease, Lipid Metabolism

Molecules in Network
ACVR1B, C14ORF139, CD3, CD36, DDX58, DYX1C1, E2f,
EFHC1, EPPK1, FST, GPAM, HAMP, HSPA1A, IFN Beta, IKK,
INHBE, INSIG2, KLF3, MAP3K8, MAP3K13, NCF2, NF- $\kappa$ B
(family), NFATC2, NF $\kappa$ B (complex), NR2F2, Rab11, RAB11B,
RAB11FIP4, RGS3, SLC2A2, SQSTM1, TFRC, TMEM126B,
TXNRD1, VSNL1

Connective Tissue Development and Function, Skeletal and Muscular System Development and Function, Tissue Morphology

Endocrine System Disorders, Hematological Disease, Metabolic Disease ADM, AGPAT9, Akt, BHLHB2, DCBLD2, HELLS, HSPG2 (includes EG:3339), Ige, IGF2, IGF2BP2, IL11, INPP5D, JAG1, Laminin, LCAT, Mek, NEXN, p70 S6k, PALLD, Pdgf, PDGF BB PP2A, Ptk, RORA, Shc, SKAP2, SLC2A3, SLC6A4, SLC6A6, SORBS2, Sos, STAT, Tgf beta, TINAG, XRN1

Adaptor protein 2, AKR1B10, Ap1, AP2A1, BNIP3L, C5ORF34, CBR1, Ck2, Creb, DENND4A, hCG, HIP1, Histone h3, HMOX1, Igfbp, IGFBP1, IGFBP3, JUN, KLF5, LDL, MAFF, MAZ, Mmp, NRP1, P38 MAPK, PFKFB3, SMC4, SPP1, SULT2A1, SYNE2, tyrosine kinase, VDR, Vegf, VitaminD3-VDR-RXR, VRK1

A1CF, AKR1C1, AKR1C2, AKR1C3, Angiotensin II receptor type 1, EGR1, ERK, ETS, F2RL1, FGFR3, Fibrin, FOS, G-Actin,

Cardiovascular System Development and Function, Tissue Morphology, Amino Acid Metabolism GCLC, GCLM, GDF15, GNRH, JUN/JUNB/JUND, KLB, KLF4, LIMA1, NF1, NGF, NTN4, PLAUR, Rar, RASGRF2, Rxr, SCLT1, SERPINE1, SWI-SNF, T3-TR-RXR, Thyroid hormone receptor, TRA2A, Trans-1,2-dihydrobenzene-1,2-diol dehydrogenase 14-3-3, ACTA1, ALB, ARHGAP26, ARHGDIA, ATP6V0A1,

Cellular Assembly and Organization, Cellular Function and Maintenance, Nervous System Development and Function ATYPICAL PROTEIN KINASE C, CCAR1, CPLX1, F Actin, F2RL2, FGD4, GABBR1, Gsk3, Insulin, Jnk, MAP2K1/2, MARCKS (includes EG:4082), NEDD4L, Nfat, PARD3, PCYT1B, Pkc(s), Pld, PRLR, Rac, Raf, Ras, Ras homolog, RGNEF, SH3BP2, SOS1, TCR, TNS1, VAMP2

ADCY, AIM1 (includes EG:202), ALP, ARFGEF1, ARID1A, Calmodulin, Calpain, Cyclin A, Cyclin E, CYP1A1, ERK1/2, G alphai, Hdac, Histone h4, IL8, IL12, Interferon alpha, ITPR2, KNG1 (includes EG:3827), Mapk, MED13, MYLK, NDRG1, NRD1, PDZK1, Pka, Pkg, PRKAR1A, PTRF, Rb, RBL1, RNA polymerase II, SMARCA4, SUV420H1, THRB

AASS, ANXA3, APPBP2, beta-estradiol, BICD1, BUB1, CCAR1, CHM, CORO2A, DIMT1L, EID3, FCGRT, geranylgeranyl pyrophosphate, GJB2, GRM3, HELLS, HRAS, IGFBP1, IREB2, Cancer, Cell Death, Reproductive System Disease KIAA2018, KLHDC2, MIRN29B2, MYC, NME5, NR3C1, PDK1, PTP4A2, RAB6A, RABGGTB, RASA4 (includes EG:10156), RPN2, SARDH, SELENBP1, SGK3, TXN

ABAT, ADM, ALDH5A1, BMP15, CDKN2B, CEACAM5 (includes EG:1048), CENPE, CENPF, CMAS, CRK, FOXG1, FSH, FST, GCLC, GPRC5A, GRLF1, HNRPDL, INHA, KCNA5, KLF10, LACTB, LYVE1, MST1R, MTMR10, PCF11, PGRMC1, RB1, SEMA6B, SMAD5, SPAG4, TGFB1, THOC1, THOC2, thyroid hormone, TRIP11

ACAP1, ADAM12, BBS7, C10ORF88, C16ORF57, C3ORF63, CALR, CD59, CLTC, CMIP, CUGBP1, DCP1A, F2RL2,

Cell Death, Cancer, Cell Cycle KIAA1219, KIAA1429, KRT18, METTL7A, MME, PDIA2, PDIA3, PHACTR2, PPP1CA, PRLR, SH3BP4, SLC6A8, SMAD4, SRC, SSB, TFE3, TNS1, TOM1, TOM1L1, TUSC3, YWHAZ, ZFYVE16 ATP5S, C3, CTBP1, CYP4B1, CYP4F11, EDA2R, ELK3, FGD4, FRY, GDF15, GPRC5A, LEFTY2, LRBA, MAP3K13, MAPK8, MAPK9, MINK1, MSLN, PEX1, PKP2, PRAM1, RAE1, RCOR3 (includes EG:55758), retinoic acid, RGL4, SH3BP5, SH3RF2, SMOX, SPAG9, SSBP3, STMN3, TAL1, TFF2, YWHAG, ZNF217
Cancer, Hepatic System Disease, Liver Hyperplasia/Hyperproliferation 


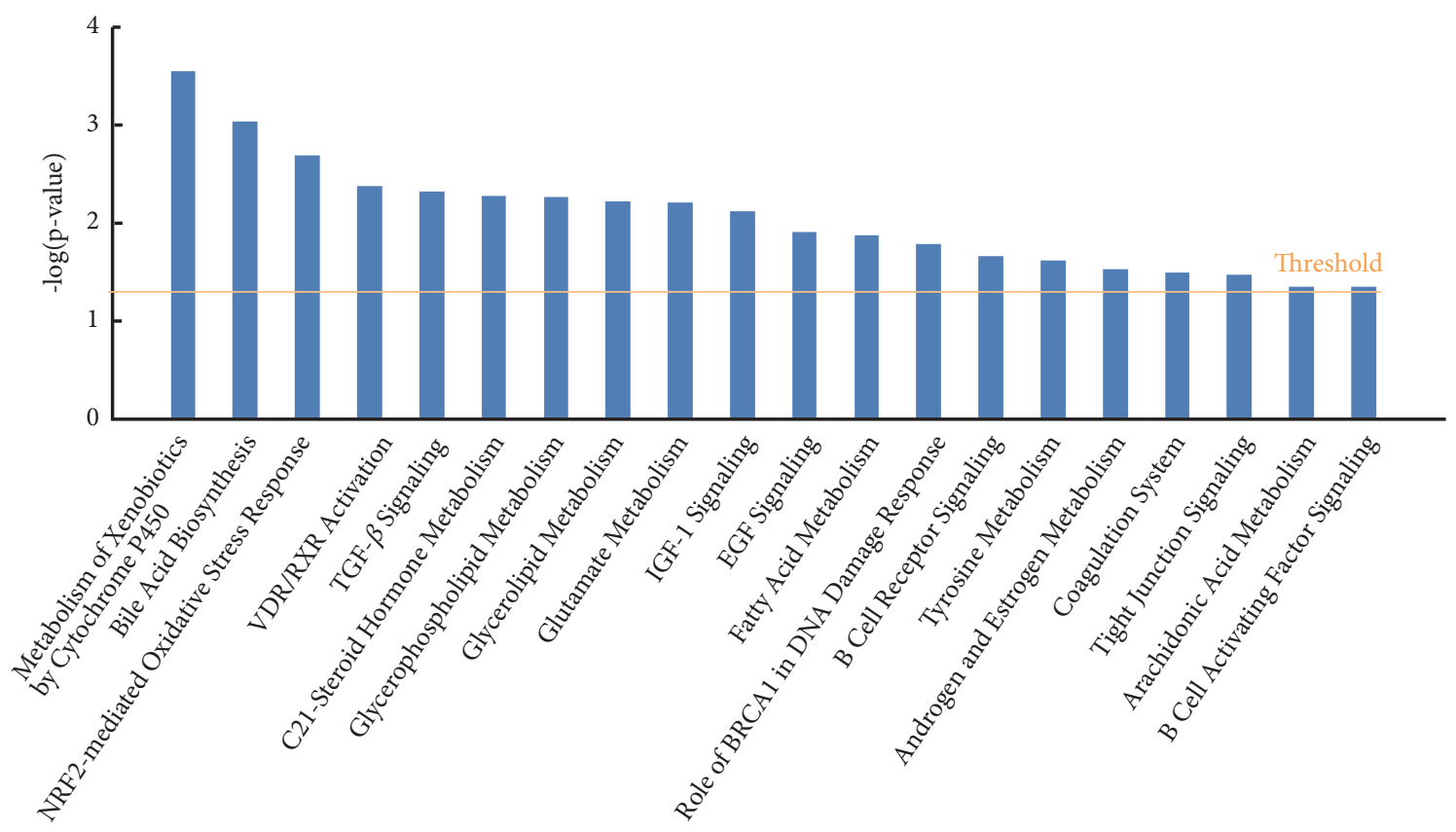

FIGURE 1: Top canonical pathway analysis of myricetin-treated HepG2 cells by IPA.

In addition to cellular function and disease, IPA system also listed out the significantly disturbed genes by distinct canonical pathways based on IPKB. As shown in Figure 1, IPA system outputs all the significantly affected signaling pathways by myricetin treatment with a $p$-value less than 0.05 . Among the total 20 canonical pathways, Nrf-2-mediated oxidative stress response, TGF- $\beta$ signaling, and B cell receptor signaling are strongly associated to chronic inflammation; metabolism of xenobiotics by cytochrome P450, bile acid biosynthesis, C21-steroid hormone metabolism, glycerophospholipid metabolism, glycerolipid metabolism, glutamate metabolism, IGF-1 signaling, and fatty acid metabolism are closely linked to metabolic disease, especially glucolipid metabolism dysfunction.

\subsection{Network Analysis of Myricetin-Treated HepG2 Cells by} $I P A$. Furthermore, IPA further built gene networks to connect key genes and enrich categories of diseases and functions, via the construction of the correlation between the significantly disturbed genes by myricetin treatment. We have listed out the top 1 network to further elucidate the cellular functions vividly and distinctly, corresponding to the top 1 cellular function and disease, as shown in Table 3.

As shown in Figure 2, top 1 network is related to cardiovascular disease, metabolic disease, and lipid metabolism. It consists of 35 genes, 28 of which are expressed differentially. It is worth noting that the cellular function of oxidative stress and inflammation response were vastly involved in this network. Among these genes, SLC2A2 (solute carrier family 2 member 2), also called GLUT2, is the most downregulated gene, with the reduction of 4.89 -fold by myricetin treatment, and its expression is indirectly adjusted by $N F-\kappa B$ family. The reduction of $S L C 2 A 2$ expression suggested the inhibition of glucose absorption, which has a strong correlation to the glucose and lipid metabolic pathways [29]. Clinical data shows that SLC2A2 (GLUT2) mutations are the cause for Fanconi-Bickel syndrome, a rare autosomal recessive disease about carbohydrate metabolism dysfunction, and the tested patients showed typical characteristics such as glycogen storage disorders and proximal renal tubular nephropathy [30]. The most upregulated gene is NCF2 (solute carrier family 2 member 2), with the induction of 4.27 -fold by myricetin treatment, and it is also indirectly adjusted by $N F-\kappa B$ family. NCF2 is reported to be associated with chronic disease, especially inflammatory chronic granulomatous disease [31]. Besides, multiple protein kinase genes related to inflammation are directly regulated by myricetin via modulation of inflammatory transcription factors. For instance, MAP3K13 and $M A P 3 K 8$, the upstream protein kinases of $\mathrm{NF}-\kappa \mathrm{B}$ pathway, displayed the decreasing expression folds of 2.06 and 2.33, respectively, where the expression of $M A P 3 K 8$ was indirectly affected by $N F-\kappa B$. Thus, we may speculate that inflammatory signal molecular and the upstream protein kinase would interact with each other.

What is more, $N F-\kappa B$ is the core of this network and monitors the activities of a series of inflammatory factors and the expressions of several typical antioxidant genes. Inflammatory genes CD36 (3.25-fold), KLF3 (2.57fold), NFATC2 (2.36-fold), and ACVR1B (2.15-fold) were downregulated indirectly by myricetin treatment via $N F$ $\kappa B$ pathway, while antioxidant genes TXNRD1 (2.04-fold), SQSTM1 (2.45-fold), TMEM126B (2.26-fold), and HSPA1A (3.35-fold) were upregulated. All the above changes imply that myricetin could inhibit inflammation and activate the antioxidant chemoprevention to play a critical role in the cellular redox equilibrium. Two typical signaling pathways 

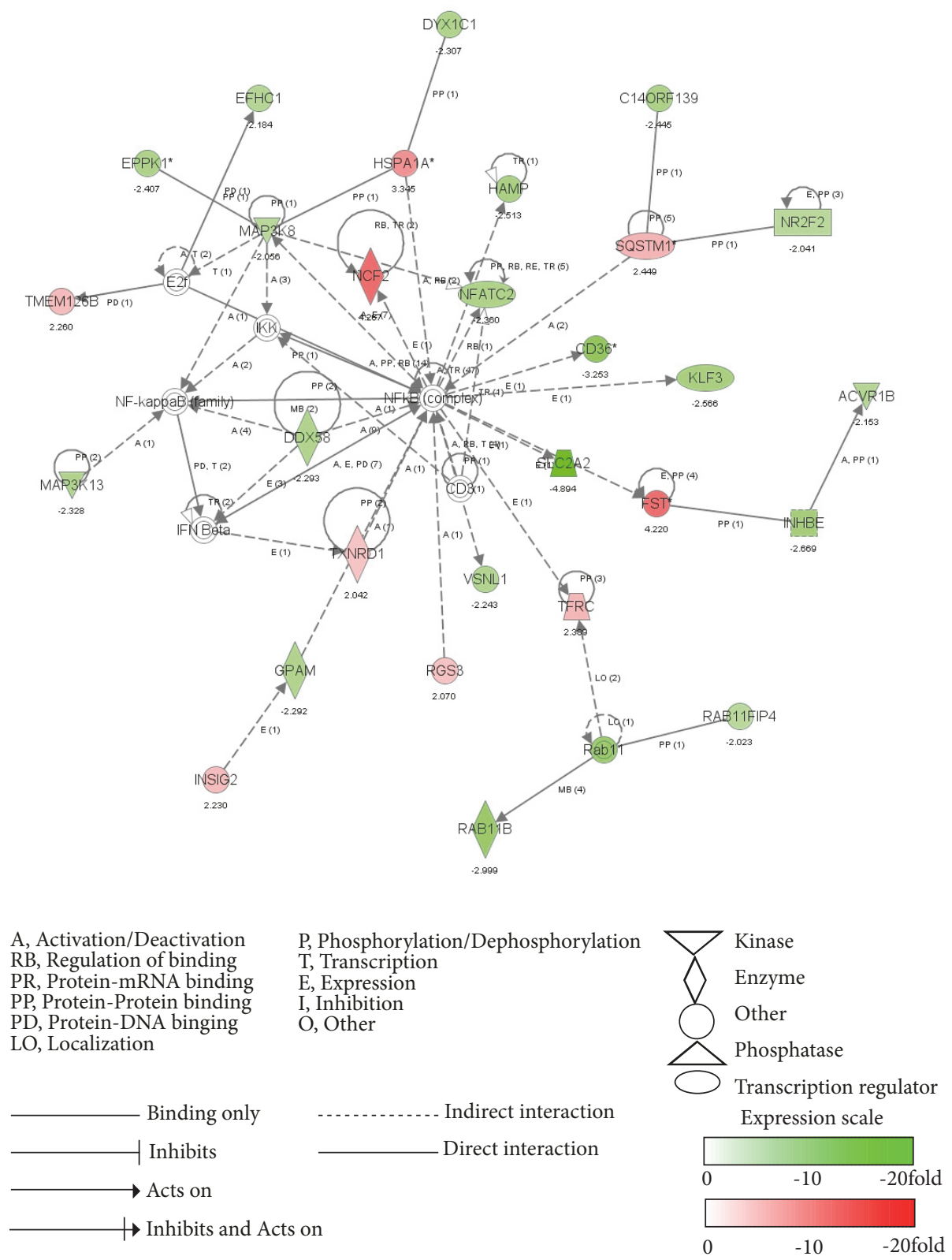

FIGURE 2: Top network analysis of myricetin-treated HepG2 cells by IPA. The dataset was analyzed by Ingenuity Pathway Analysis software. The node color indicates the expression level of the genes. Nodes and edges are displayed with various shapes and labels that present the functional class of genes and the nature of the relationship between the nodes, respectively.

are found to be involved in the top 1 network, including $B$ cell receptor signaling and Nrf2-mediated antioxidant pathway. B cell receptor signaling was reported to activate NF- $\kappa$ B by PKC/TAK1 pathway [32]. NFATC2, MAP3K13, and $M A P 3 K 8$ are belonging to $\mathrm{B}$ cell receptor signaling, and their downregulation reveals that myricetin can exert its antiinflammatory effect via inhibition of B cell receptor signaling. Similarly, the upregulation of SQSTM1 and TXNRD1 in Nrf2mediated antioxidant pathway indicates that myricetin can also inhibit inflammation via activation of Nrf2-mediated antioxidant pathway.
3.5. Real-Time qPCR Verification of the Specific Functional Genes. To verify the microarray data and the above biofunction caused by myricetin in HepG2 cells, we performed RTqPCR and compared the result of selective top 20 altered molecules in IPA to the raw gene chip data. As shown in Figure 3(a), we have selected typical significantly disturbed genes with their respective change fold from microarray data by myricetin treatment, including antioxidant genes GCLC, AKR1C1/2/3 and NCF2, inflammatory genes SERPINE, ARHGAP26, FST and IL11, and metabolic genes IGFBP1, F2RL2, SLC2A2/6A6, and HSPA1A, which are all 


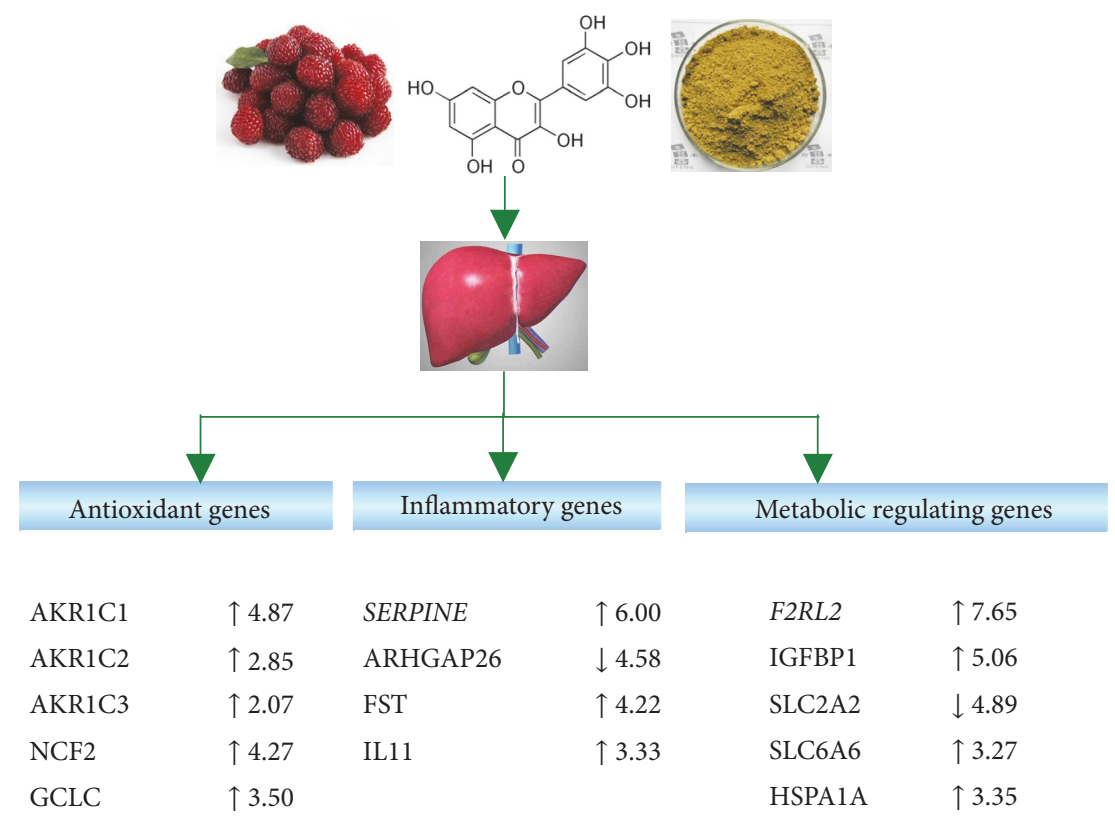

(a)
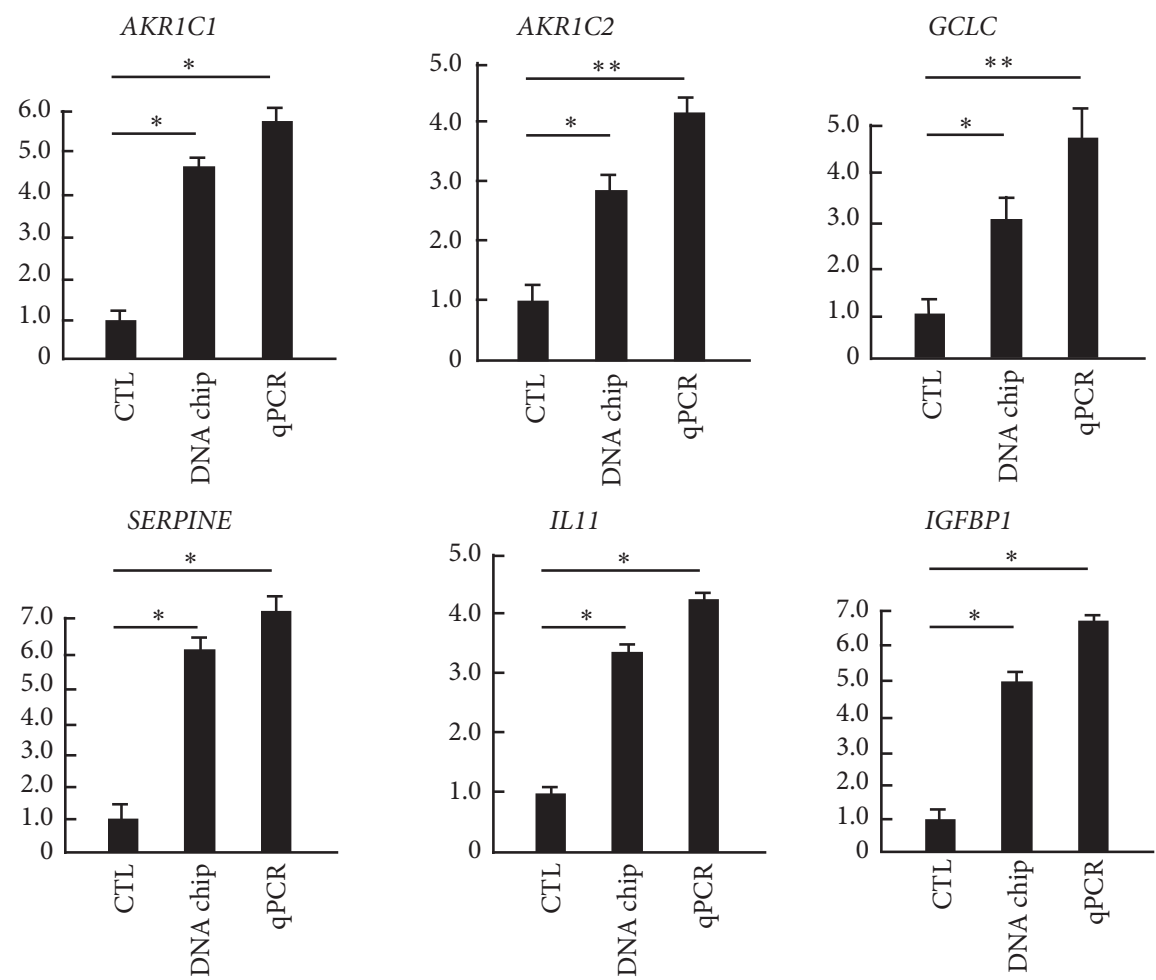

(b)

FIGURE 3: Real-time quantitative PCR verification of the specific functional genes identified by DNA microarray and IPA. (a) Three basic biofunction related genes filtrated by IPA. After DNA microarray, the whole data were input into ingenuity pathway analysis system. The genes classified to antioxidant genes, inflammatory genes, and metabolic regulating genes are displayed with more than twofold expression change by myricetin. (b) Real-time PCR verification data versus microarray data. HepG2 cells were pretreated with or without myricetin for $9 \mathrm{~h}$. RNA extract and real-time PCR were described in Material and Methods. The result was expressed as the relative expression level. Each value represents the mean \pm SD of three separate experiments, $* \mathrm{p}<0.05 ; * * \mathrm{p}<0.01$ versus control, respectively. CTL, control; AKR1C, aldo-keto reductase family 1, member C; GCLC, glutamate cysteine ligase, catalytic subunit; SERPINE, serpin peptidase inhibitor clade; IL, interleukin; IGFBP1, insulin -like growth factor-binding protein 1. 
regulated by Nrf2 or NF- $\kappa \mathrm{B}$ signaling pathways. Then, we further verify the gene chip results of several selected key genes by RT-qPCR and make a comparison. As shown in Figure 3(b), RT-qPCR results have confirmed the accuracy of the gene chip results, and their changes are even bigger. These results indicate that myricetin can stimulate the expressions of antioxidant genes, inhibit that of inflammatory genes, and induce the expressions of metabolic regulating genes in HepG2 cells.

3.6. Bio-Plex Based Inflammatory Cytokines Assay by Myricetin Treatment in HepG2 Cells Induced by LPS. The above IPA analysis results reveal that inflammation is the main interface targeted by myricetin. However, these results just stay in mRNA level. To investigate whether myricetin indeed works on inflammation inhibition, and we conducted Western blotting and bio-plex inflammatory cytokine assay in HepG2 cells, which is a more suitable inflammation model. Cytokines are important in cell signaling and play a significant role in inflammation [33].

Before starting, we firstly performed Western blotting of typical biomarkers of inflammation, iNOS and COX2 , to confirm the anti-inflammatory efficiency and the toxicity of myricetin. The results showed that iNOS and COX-2 expressions are both significantly decreased dosedependently (Figure $4(\mathrm{a})$ ) and $20 \mu \mathrm{M}$ of myricetin had no cytotoxicity on HepG 2 cells, which is similar to our previous study[18]. Then, we applied 10-20 $\mu \mathrm{M}$ of myricetin in bioplex cytokines assay. As shown in Figure 4(b), 27 kinds of cytokines were detected by bio-plex system, just 9 of which were significantly inhibited by myricetin pretreatment, including TNF- $\alpha$, IFN- $\gamma$, IL- $1 \alpha$, IL-1 $\beta$, IL-2, and IL-6.

\section{Discussion}

In the present study, we conducted microarray and bioinformatic analysis to comprehensively describe the biofunction of myricetin in HepG2 cells, GO, and IPA analysis results provided some hints for the anti-inflammation, antioxidation, and intervention on metabolism of myricetin, which were partly verified by RT-qPCR. The function and canonical pathway analysis by IPA further confirmed that myricetin plays a crucial role on the regulation of redox signaling pathways and metabolic process, especially the inhibition on inflammation. Thus, we finally performed the proteomic analysis by the application of bio-plex cytokines assay, and the results indicated that myricetin does exert potent antiinflammatory effect by secretion inhibitions of IL- $1 \alpha$, IL- $1 \beta$, IL-2, IL-6, IFN- $\gamma$, and TNF- $\alpha$.

Top molecules generated by IPA help us to focus on the most impossible biofunction rapidly. For example, top metabolic gene SLC2A2 plays a key role in HIF-1- $\alpha$ signaling, which acts as the central system on regulation of metabolism. The concentration of HIF-1- $\alpha$ (and its subsequent activity) is regulated by NF- $\kappa \mathrm{B}$-dependent regulation [34]. HIF-1 is overexpressed in many human cancers, and its overexpression promotes tumour growth and metastasis through its initial angiogenesis and regulation of cell metabolism to overcome hypoxia [35]. Thus, an inference can be made from the phenomenon that SLC2A2 was involved in the HIF-1- $\alpha$ signaling, and its decreased expression by myricetin treatment implies that myricetin has the potency on the regulation of metabolic process, which is evidenced by the canonical pathway analysis of IPA. As shown in Figure 1, several metabolic pathways have been found to be associated with the metabolic regulation property of myricetin, including "metabolism of xenobiotics by cytochrome P450, bile acid biosynthesis, C21-steroid hormone metabolism, glycerophospholipid metabolism, glycerolipid metabolism, glutamate metabolism, IGF-1 signaling, and fatty acid metabolism." These metabolic pathways were all significantly disturbed by myricetin treatment, indicating that myricetin possesses the ability of regulating glucolipid metabolism dysfunction.

In addition to the top 1 network associated with cellular function and disease, top 2 and top 3 networks are also found to be related to inflammation. As shown in Table 3 , the top 2 and top 3 cellular functions and diseases are "connective tissue development and function, skeletal and muscular system development and function, and tissue morphology" (with a score of 33 and focus molecules of 22) and "endocrine system disorders, haematological disease, and metabolic disease" (with a score of 32 and focus molecules of 22). The cores of their networks are TGF- $\beta$ and AP-1 (data not shown here), and their respective key molecules include IL11, STAT, SORBS2, Sos, NEXN, SLC6A4, $X R N 1$, etc. Most of the inflammatory genes were significantly downregulated by myricetin treatment; however, a series of antioxidant genes, such as SQSTM1, TXNRD1, HMOX1, and SLC family, were found to be upregulated significantly, which reveals that myricetin plays a complex role on the balance between inflammation and antioxidation, the redox interface in HepG2 cells. Moreover, glucolipid metabolism was also found to be related to the redox status, for the disturbed expressions of IGF2, IGF2BP2, IGFBP1, and IGFBP3.

Our study is the first time to apply the inflammatory proteomic tool of bio-plex cytokines assay to investigate the inflammatory property of myricetin in HepG 2 cells. In the total typical 27 cytokines, the secretions of IL- $1 \alpha$, IL- $1 \beta$, IL2 , IL- 6 , IFN- $\gamma$, and TNF- $\alpha$ have been found to be inhibited by myricetin treatment. TNF- $\alpha$, acting as a major member of tumour necrosis factor, is an important proinflammatory cytokine in the inflammatory response model. TNF- $\alpha$, by means of binding to its receptor, can regulate NF- $\kappa \mathrm{B}$ expression by modulation of TRAF2 and RIP [36]. Therefore, the significant decrease of TNF- $\alpha$ expression indicates that the remission of inflammatory response is caused by myricetin treatment through the inhibition of NF- $\kappa \mathrm{B}$ activity, which is evidenced by other studies [37]. IL-6 and IL-1 are also important proinflammatory cytokines, and they were found to be inhibited remarkably by myricetin treatment in the present study. As reported, LPS can activate NF- $\kappa \mathrm{B}$ by stimulating monocyte MAPK signaling pathway and then promote the expression of IL-1 gene; besides, IL-1 can induce activation of $\mathrm{p} 38$ to promote the expression of NF- $\kappa \mathrm{B}$ in turn [38]. IL-6 is closely correlated with STAT3, and STAT3 plays a key role in cell proliferation and differentiation, so IL-6 can not only promote the development of inflammation but 

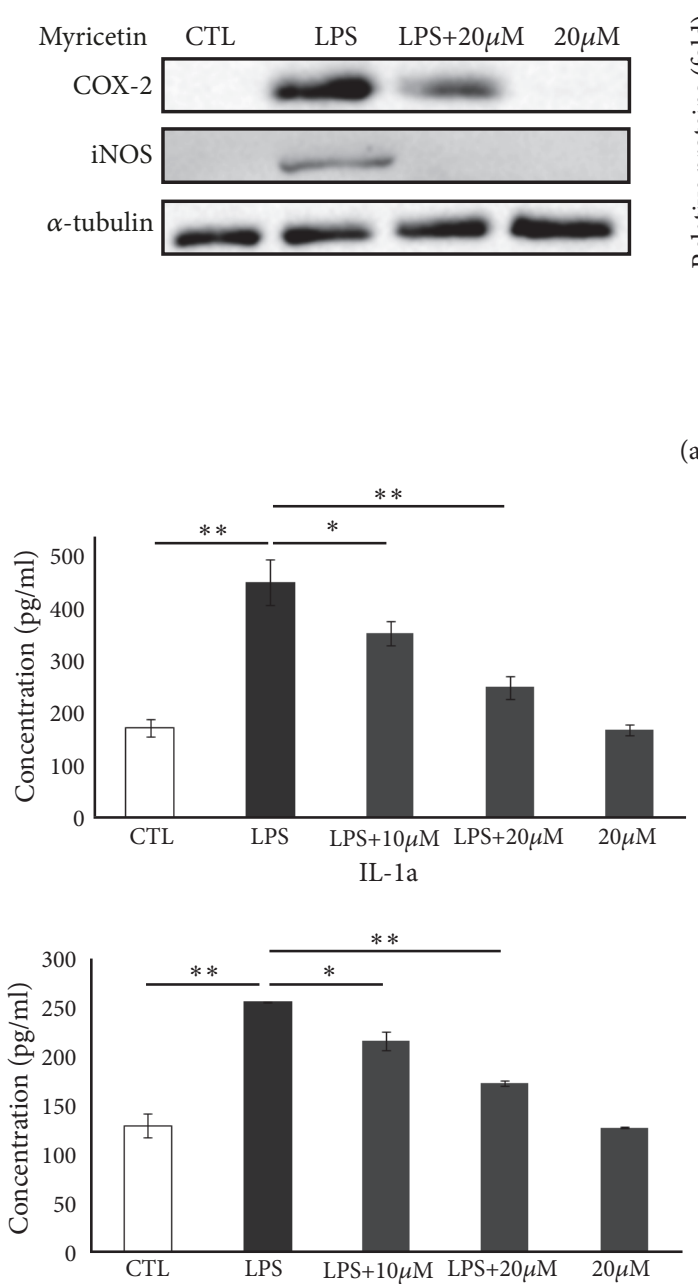

IL-2

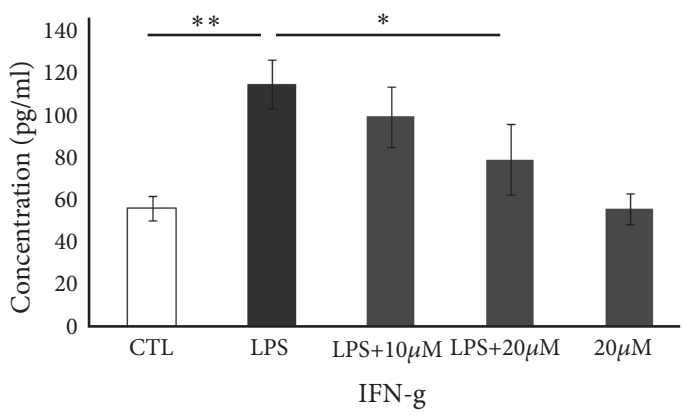

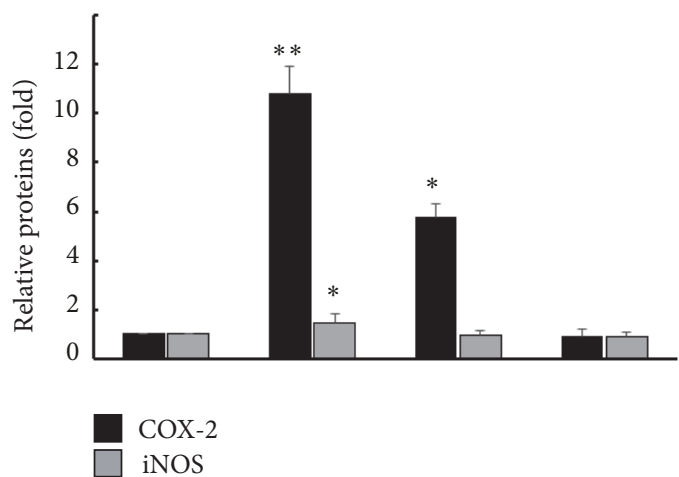

(a)
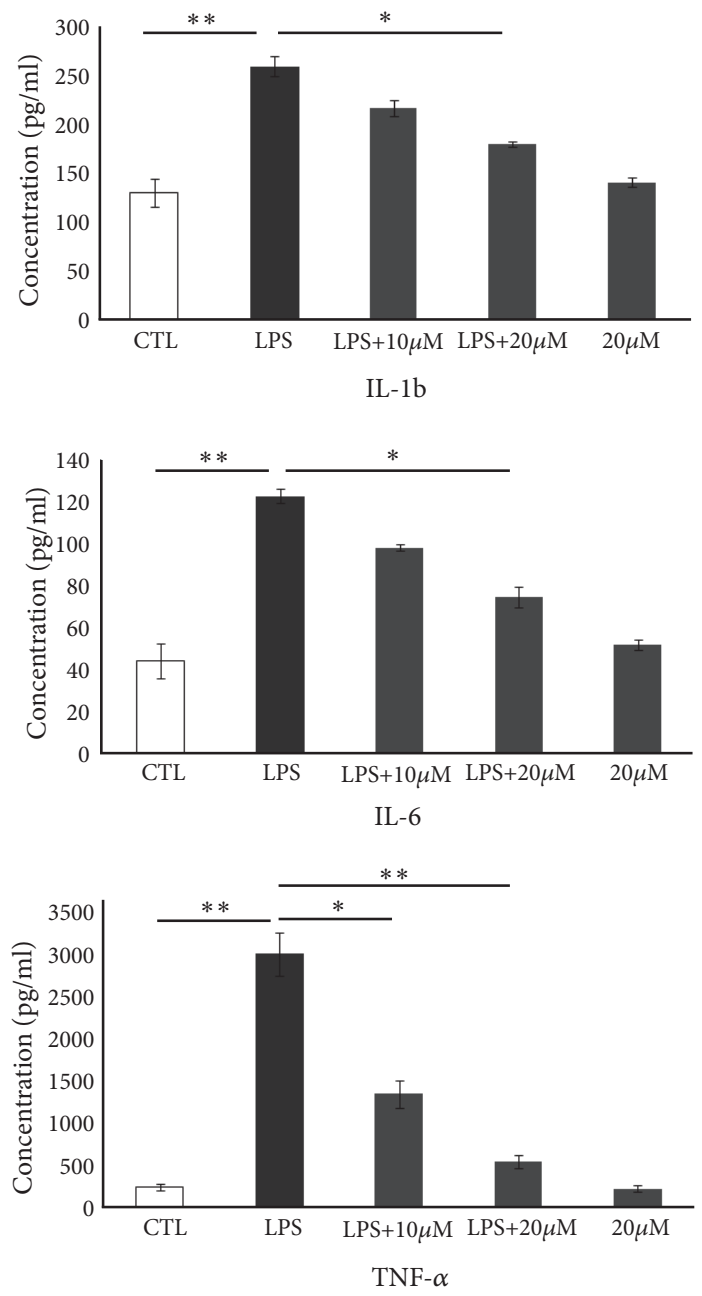

(b)

FIGURE 4: Influence of myricetin on the production of inflammatory proteins and cytokines. (a) Influence of myricetin on the production of iNOS and COX-2 protein. HepG2 cells (1×106 cells) were precultured for $24 \mathrm{~h}$ and starved in serum-free medium for $2.5 \mathrm{~h}$. The cells were then treated with the indicated concentrations of myricetin for $30 \mathrm{~min}$ and then exposed to $1 \mu \mathrm{g} / \mathrm{mL}$ LPS for $12 \mathrm{~h}$. The proteins of iNOS, COX-2, and $\alpha$-tubulin were detected by Western blotting with their antibodies, respectively. (b) Myricetin decreased the levels of multiple inflammatory cytokines in HepG2 cells. The levels of 27 kinds of cytokines were measured by multiplex technology and bio-plex assay and arranged in an order from high to low change in the experimental inflammatory HepG2 cells. The data represent mean \pm SD of four mice. $* \mathrm{p}<0.05$ and $* * \mathrm{p}<0.01$. CTL, control; IL, interleukin; IFN- $\gamma$, interferon-gamma; TNF- $\alpha$, tumor necrosis factor. 
also accelerate the proliferation of normal cells and tumour cells [39]. Hence, NF- $\kappa$ B signaling in the HepG2 cells was inhibited by myricetin because of the decrease of IL- 6 and IL1 productions. According to the result of proteomic assay of cytokines, spontaneously, the speculation that myricetin has a powerful effect on the inhibition of inflammation is obtained, which encourages in vivo studies to verify its possible health benefits.

\section{Conclusions}

Obviously, by the combined application of genomic microarray and proteomic cytokine assay, we not only comprehensively understand the multiple functions of myricetin, in the form of regulation on redox signaling pathways and metabolic process at mRNA level, but also verify its crucial and potent anti-inflammatory property at protein level. Therefore, myricetin exerts potent multiple biofunctions linked to redox balance and metabolic regulation, especially its remarkable anti-inflammatory activity. The present study could promote the application of nutritional intervention by myricetin in the research and development of functional food or special medical use food.

\section{Abbreviations}

AP-1: Activator protein 1

GF: $\quad$ Growth factor

GO: Gene Ontology

$\mathrm{I} \kappa \mathrm{B}: \quad$ Inhibitory protein of NF- $\kappa \mathrm{B}$

IRF3: Interferon regulatory factor 3

IL: Interleukin

IFN: Interferon

IPA: Ingenuity pathways analysis

LPS: Lipopolysaccharides

MAPK: Mitogen-activated protein kinase

NF- $\kappa$ B: Nuclear factor-kappa B

Nrf2: $\quad$ Nuclear factor-erythroid 2 p45-related factor 2

NCF2: Neutrophil cytosolic factor $2(65 \mathrm{kDa}$, chronic granulomatous disease, autosomal 2)

RT-PCR: Real-time quantity polymerase chain reaction

SLC2A2: Solute carrier family 2 (facilitated glucose transporter), member 2

TGF- $\beta$ : Transforming growth factor beta

TNF: Tumour necrosis factor.

\section{Data Availability}

The data used to support the findings of this study are available from the corresponding author upon request.

\section{Disclosure}

We have just submitted the abstract and give an oral presentation with some of the content in this manuscript in the $3 \mathrm{rd}$
International Conference on Agriculture and Agro-Industry 2018 (ICAAI2018), but without publishing this manuscript. Tao Li and Jihe Zhu are joint first authors.

\section{Conflicts of Interest}

The authors declare that there are no conflicts of interest regarding the publication of this paper.

\section{Authors' Contributions}

Tao Li and Jihe Zhu contributed equally to the work.

\section{Acknowledgments}

This work was partially supported by Natural Science Foundation of China and Hunan Province, Key Scientific Research Fund of Hunan Provincial Science and Technology Department (2017NK2093), Core Research Program 1515 and double first-class construction project of Hunan Agricultural University (No. SYL201802025) to Si Qin. The authors would like to thank Key Laboratory for Food Science and Biotechnology of Hunan Province, College of Food Science and Technology, Hunan Agricultural University for providing the facilities to carry out this study.

\section{Supplementary Materials}

Supplementary Table 1: the disturbed genes involved in signal transduction. Supplementary Table 2: the disturbed genes involved in nucleus and mitochondrion. Supplementary Table 3: the disturbed genes involved in DNA binding, protein binding, kinase, and protein kinase activity. (Supplementary Materials)

\section{References}

[1] J. M. Harnly, R. F. Doherty, G. R. Beecher et al., "Flavonoid content of U.S. fruits, vegetables, and nuts," Journal of Agricultural and Food Chemistry, vol. 54, no. 26, pp. 9966-9977, 2006.

[2] K. C. Ong and H. E. Khoo, "Biological effects of myricetin," General Pharmacology: The Vascular System, vol. 29, no. 2, pp. 121-126, 1997.

[3] K. W. Lee, N. J. Kang, E. A. Rogozin et al., "Myricetin is a novel natural inhibitor of neoplastic cell transformation and MEK1," Carcinogenesis, vol. 28, no. 9, pp. 1918-1927, 2007.

[4] P. K. Maurya, P. Kumar, S. Nagotu, S. Chand, and P. Chandra, "Multi-target detection of oxidative stress biomarkers in quercetin and myricetin treated human red blood cells," RSC Advances, vol. 6, no. 58, pp. 53195-53202, 2016.

[5] Y. Qiu, N. Cong, M. Liang, Y. Wang, and J. Wang, "Systems pharmacology dissection of the protective effect of myricetin against acute ischemia/reperfusion-induced myocardial injury in isolated rat heart," Cardiovascular Toxicology, vol. 17, no. 3, pp. 277-286, 2017.

[6] R. Domitrović, K. Rashed, O. Cvijanović, S. Vladimir-Knežević, M. Škoda, and A. Višnić, "Myricitrin exhibits antioxidant, antiinflammatory and antifibrotic activity in carbon tetrachlorideintoxicated mice," Chemico-Biological Interactions, vol. 230, pp. 21-29, 2015. 
[7] B. O. Cho, H. H. Yin, S. H. Park, E. B. Byun, H. Y. Ha, and S. I. Jang, "Anti-inflammatory activity of myricetin from diospyros lotus through suppression of $\mathrm{nf}-\kappa \mathrm{b}$ and statl activation and nrf2-mediated ho-1 induction in lipopolysaccharidestimulated raw264.7 macrophages," Bioscience, Biotechnology, and Biochemistry, vol. 80, no. 8, pp. 1520-1530, 2016.

[8] F. Sun, X. Y. Zheng, J. Ye, T. T. Wu, J. L. Wang, and W. Chen, "Potential anticancer activity of myricetin in human T24 bladder cancer cells both in vitro and in vivo," Nutrition and Cancer, vol. 64, no. 4, pp. 599-606, 2012.

[9] X.-H. Zhang, S.-Y. Chen, L. Tang et al., "Myricetin induces apoptosis in HepG2 cells through Akt/p70s6K/Bad signaling and mitochondrial apoptotic pathway," Anti-Cancer Agents in Medicinal Chemistry, vol. 13, no. 10, pp. 1575-1581, 2013.

[10] M. Müller and S. Kersten, "Nutrigenomics: goals and strategies," Nature Reviews Genetics, vol. 4, no. 4, pp. 315-322, 2003.

[11] K. R. Martin, "Using nutrigenomics to evaluate apoptosis as a preemptive target in cancer prevention," Current Cancer Drug Targets, vol. 7, no. 5, pp. 438-446, 2007.

[12] J. Wang, X. Bai, X. Ding et al., "Quantitative proteomic analysis reveals the role of tea polyphenol EGCG in egg whites in response to vanadium stress," Nutrition Journal, vol. 39-40, pp. 20-29, 2017.

[13] L. Méndez, S. Ciordia, M. S. Fernández et al., "Changes in liver proteins of rats fed standard and high-fat and sucrose diets induced by fish omega-3 PUFAs and their combination with grape polyphenols according to quantitative proteomics," The Journal of Nutritional Biochemistry, vol. 41, pp. 84-97, 2017.

[14] F. Ali, A. Ismail, N. M. Esa, and C. P. Pei, "Transcriptomics expression analysis to unveil the molecular mechanisms underlying the cocoa polyphenol treatment in diet-induced obesity rats," Genomics, vol. 105, no. 1, pp. 23-30, 2015.

[15] S. Malireddy, S. R. Kotha, J. D. Secor et al., "Phytochemical antioxidants modulate mammalian cellular epigenome: implications in health and disease," Antioxidants \& Redox Signaling, vol. 17, no. 2, pp. 327-339, 2012.

[16] W. Zam and A. Khadour, "Impact of phytochemicals and dietary patterns on epigenome and cancer," Nutrition and Cancer, vol. 69, no. 2, pp. 184-200, 2017.

[17] J.-C. Wu, C.-S. Lai, P.-S. Lee et al., "Anti-cancer efficacy of dietary polyphenols is mediated through epigenetic modifications," Current Opinion in Food Science, vol. 8, pp. 1-7, 2016.

[18] S. Qin, J. Chen, S. Tanigawa, and D.-X. Hou, "Microarray and pathway analysis highlight Nrf2/ARE-mediated expression profiling by polyphenolic myricetin," Molecular Nutrition \& Food Research, vol. 57, no. 3, pp. 435-446, 2013.

[19] B. Kloesch, T. Becker, E. Dietersdorfer, H. Kiener, and G. Steiner, "Anti-inflammatory and apoptotic effects of the polyphenol curcumin on human fibroblast-like synoviocytes," International Immunopharmacology, vol. 15, no. 2, pp. 400-405, 2013.

[20] J. Chen, T. Uto, S. Tanigawa, T. Kumamoto, M. Fujii, and D.-X. Hou, "Expression profiling of genes targeted by bilberry (Vaccinium myrtillus) in macrophages through DNA microarray," Nutrition and Cancer, vol. 60, no. 1, pp. 43-50, 2008.

[21] M. F. Neurath, "Cytokines in inflammatory bowel disease," Nature Reviews Immunology, vol. 14, no. 5, pp. 329-342, 2014.

[22] J. M. Rubio-Perez and J. M. Morillas-Ruiz, "A review: inflammatory process in alzheimer's disease, role of cytokines," The Scientific World Journal, vol. 2012, Article ID 756357, 15 pages, 2012.
[23] J. Helleman, M. Smid, M. P. H. M. Jansen, M. E. L. van der Burg, and E. M. J. J. Berns, "Pathway analysis of gene lists associated with platinum-based chemotherapy resistance in ovarian cancer: the big picture," Gynecologic Oncology, vol. 117, no. 2, pp. 170-176, 2010.

[24] C.-J. Li, R. W. Li, Y.-H. Wang, and T. H. Elsasser, "Pathway analysis identifies perturbation of genetic networks induced by butyrate in a bovine kidney epithelial cell line," Functional \& Integrative Genomics, vol. 7, no. 3, pp. 193-205, 2007.

[25] N. K. Sethy, M. Singh, R. Kumar, G. Ilavazhagan, and K. Bhargava, "Upregulation of transcription factor NRF2-mediated oxidative stress response pathway in rat brain under short-term chronic hypobaric hypoxia," Functional \& Integrative Genomics, vol. 11, no. 1, pp. 119-137, 2011.

[26] M. A. Kluge, J. L. Fetterman, and J. A. Vita, "Mitochondria and endothelial function," Circulation Research, vol. 112, pp. 11711188, 2013.

[27] D. Voet, J. G. Voet, and C. W. Pratt, "Cancer cytogenetics: chromosomal and molecular genetic aberrations of tumour cells," in Journal of General Plant Pathology, vol. 70, pp. 278-283, 4th edition, 2015.

[28] D. A. Walsh and C. D. Ashby, "Protein kinases: aspects of their regulation and diversity," Recent Progress in Hormone Research, vol. 29, pp. 329-359, 1973.

[29] A. R. Saltiel and C. R. Kahn, "Insulin signalling and the regulation of glucose and lipid metabolism," Nature, vol. 414, no. 6865 , pp. 799-806, 2001.

[30] W. Wang, M. Wei, H. M. Song et al., "SLC2A2 gene analysis in three Chinese children with Fanconi-Bickel syndrome," Zhongguo Dang Dai Er Ke Za Zhi, vol. 17, pp. 362-366, 2015.

[31] M. Gentsch, A. Kaczmarczyk, K. Van Leeuwen et al., "Alurepeat-induced deletions within the NCF2 gene causing p67phox-deficient chronic granulomatous Disease (CGD)," Human Mutation, vol. 31, no. 2, pp. 151-158, 2010.

[32] Y. Herishanu, P. Pérez-Galán, D. Liu et al., “The lymph node microenvironment promotes B-cell receptor signaling, NF- $\kappa \mathrm{B}$ activation, and tumor proliferation in chronic lymphocytic leukemia," Blood, vol. 117, no. 2, pp. 563-574, 2011.

[33] G. Landskron, M. De la Fuente, P. Thuwajit, C. Thuwajit, and M. A. Hermoso, "Chronic Inflammation and Cytokines in the Tumor Microenvironment," Journal of Immunology Research, vol. 2014, Article ID 149185, 19 pages, 2014.

[34] G. Li, Y. Zhang, Y. Qian et al., "Interleukin-17A promotes rheumatoid arthritis synoviocytes migration and invasion under hypoxia by increasing MMP2 and MMP9 expression through NF- $\kappa \mathrm{B} / \mathrm{HIF}-1 \alpha$ pathway," Molecular Immunology, vol. 53, no. 3, pp. 227-236, 2013.

[35] G. L. Semenza, "HIF-1 mediates metabolic responses to intratumoral hypoxia and oncogenic mutations," The Journal of Clinical Investigation, vol. 123, no. 9, pp. 3664-3671, 2013.

[36] H. Zelová and J. Hošek, “TNF- $\alpha$ signalling and inflammation: interactions between old acquaintances," Inflammation Research, vol. 62, no. 7, pp. 641-651, 2013.

[37] C. Xie, J. Kang, Z. Li et al., “The açaí flavonoid velutin is a potent anti-inflammatory agent: Blockade of LPS-mediated TNF- $\alpha$ and IL- 6 production through inhibiting NF- $\kappa$ B activation and MAPK pathway," The Journal of Nutritional Biochemistry, vol. 23, no. 9, pp. 1184-1191, 2012.

[38] B.-P. Huang, C.-H. Lin, H.-M. Chen, J.-T. Lin, Y.-F. Cheng, and S.-H. Kao, "AMPK activation inhibits expression of proinflammatory mediators through downregulation of PI3K/p38 MAPK 
and NF- $\kappa \mathrm{B}$ signaling in murine macrophages," DNA and Cell Biology, vol. 34, no. 2, pp. 133-141, 2015.

[39] B. Tu, L. Du, Q.-M. Fan, Z. Tang, and T.-T. Tang, "STAT3 activation by IL- 6 from mesenchymal stem cells promotes the proliferation and metastasis of osteosarcoma," Cancer Letters, vol. 325 , no. 1, pp. 80-88, 2012. 


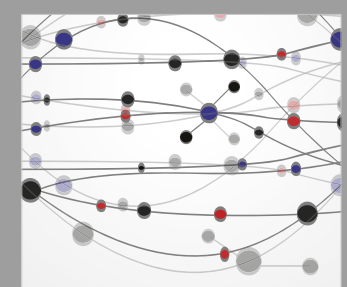

The Scientific World Journal
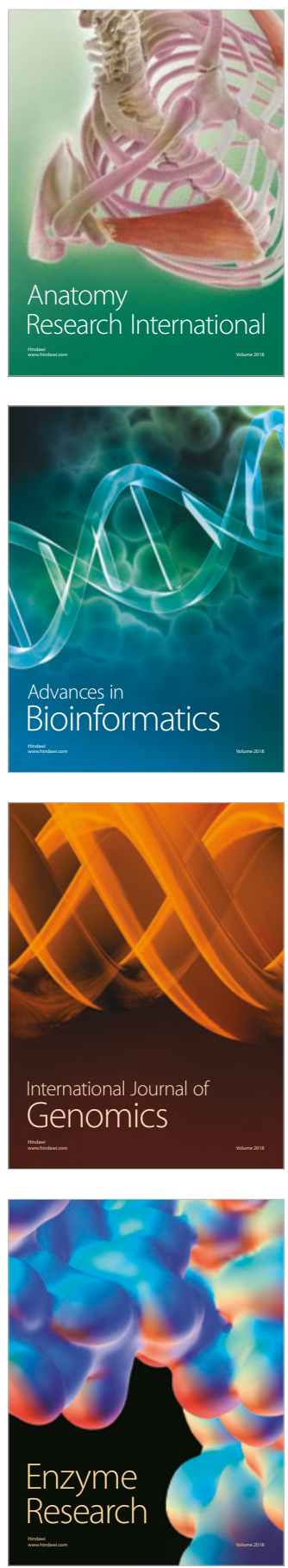
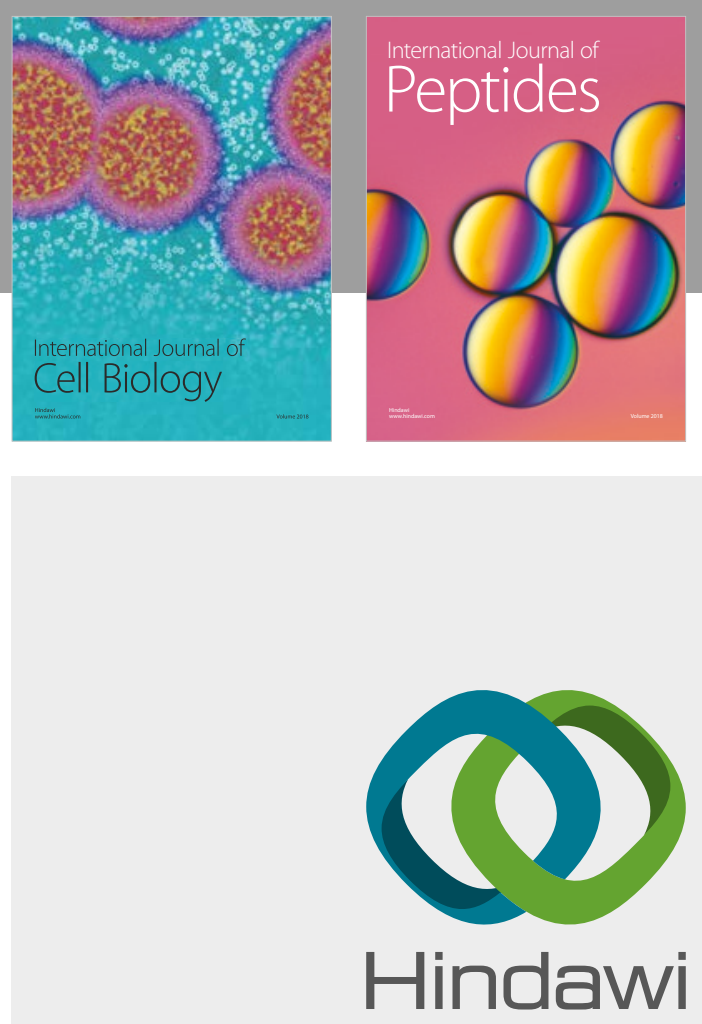

Submit your manuscripts at

www.hindawi.com
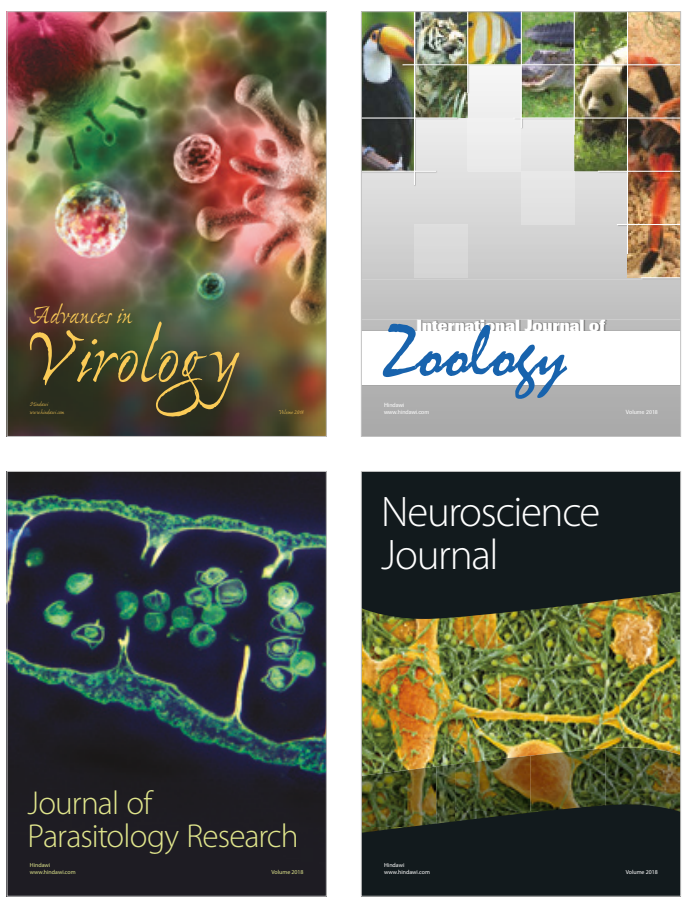
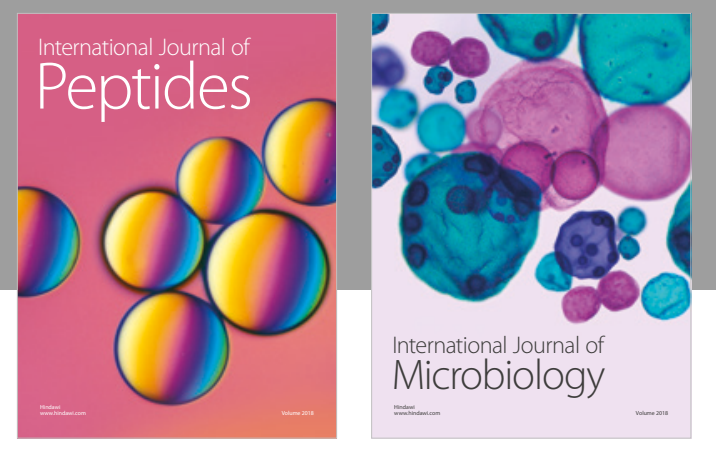

nternational Journal of Microbiology
Journal of
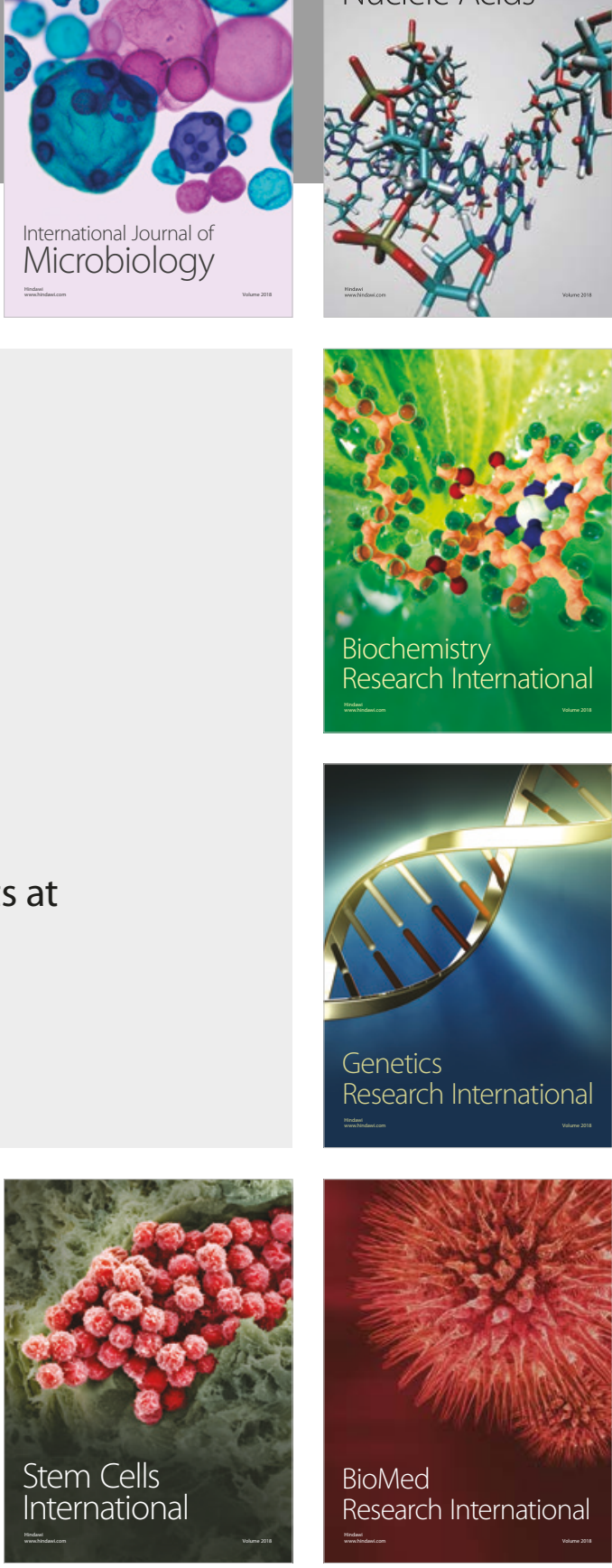
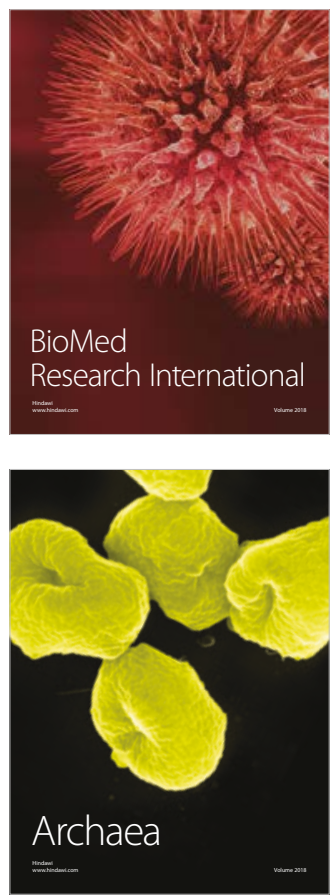